\title{
Value of Endoscopic Ultrasonography in Evaluating Unexplained Isolated Common Bile Duct Dilation on Imaging
}

\author{
Seifeldin Hakim and Amrita Sethi \\ Division of Digestive and Liver Disease Columbia University Medical Center, NY, USA
}

See "Diagnostic Value of Endoscopic Ultrasonography for Common Bile Duct Dilatation without Identifiable Etiology Detected from Cross-Sectional Imaging” by Nonthalee Pausawasdi, Penprapai Hongsrisuwan, Lubna Kamani, Kotchakon Maipang, Phunchai Charatcharoenwitthaya, on page 122-127. Clin Endosc 2022;55:47-48

Currently, there has been an increase in the number of incidental findings encountered. This may be attributed to several factors such as the widespread use of various abdominal imaging modalities, improved imaging quality, and increased clinician awareness. One such finding is a dilated common bile duct (CBD) without definitive etiology detected on transabdominal ultrasound (TUS), computed tomography (CT), or magnetic resonance cholangiopancreatography (MRCP). In addition to radiological evidence, dilated CBD can be associated with abdominal symptoms and/or abnormal liver chemistries. Various factors can alter CBD size, including benign or malignant obstruction, choledochal cysts, age, previous surgical interventions, and narcotic use. Despite controversies regarding the cutoff for normal CBD, a diameter of $7 \mathrm{~mm}$ is widely accepted as the upper limit of normal in patients without a history of cholecystectomy and $10 \mathrm{~mm}$ in diameter in post-cholecystectomy patients. ${ }^{1-3}$

Currently available abdominal imaging techniques exhibit good sensitivity and specificity in detecting biliary tract diseases. However, there are still limitations in the analysis of

Received: December 20, 2021 Revised: January 7, 2022

Accepted: January 8, 2022

Correspondence: Amrita Sethi

Columbia University Medical Center, 630 W 168th St , P\&S, 3-401, New York, NY 10032, USA

Tel: +1-212-305-1909, Fax: +1-212-305-1081, E-mail: asethimd@gmail.com ORCID: https://orcid.org/0000-0001-7989-0242

(c) This is an Open Access article distributed under the terms of the Creative Commons Attribution Non-Commercial License (http://creativecommons.org/ licenses/by-nc/3.0) which permits unrestricted non-commercial use, distribution, and reproduction in any medium, provided the original work is properly cited. small stones, intraductal stones and ampullary lesions, and in the case of CT, tumors less than $2 \mathrm{~cm}$ in size. ${ }^{2}$ MRCP provides a very high sensitivity and specificity in detecting biliary tract diseases, including stones and biliary tumors, but sensitivity decreases in stones $\geq 3 \mathrm{~mm}$. ${ }^{3}$ Over the past two decades, endoscopic ultrasound (EUS) has played an increasingly significant role in the evaluation of biliary duct dilation of unclear etiology. ${ }^{1}$ Studies have revealed comparable diagnostic accuracy between EUS and MRCP, but the diagnostic performance of MRCP declines in cases of dilated CBD, small stones, and small ampullary lesions. ${ }^{4}$ Their high diagnostic value has led to these modalities becoming the preferred primary steps prior to performing more invasive procedures such as endoscopic retrograde cholangiopancreatography (ERCP). Doing so prevents the occurrence of risks such as post-ERCP pancreatitis, bleeding, or perforation. Despite the frequent use of EUS in this diagnostic dilemma, its role and diagnostic value have not yet been demonstrated. ${ }^{4}$

In this issue of Clinical Endoscopy, Pausawasdi et al. ${ }^{5}$ evaluated the diagnostic performance of EUS in both symptomatic and asymptomatic patients with dilated CBD with no known etiology on prior imaging. Amongst 131 patients, an etiology was detected by EUS in $67 \%$. Surprisingly, the most frequent reported pathology was malignant obstruction $(31 \%)$, followed by choledocholithiasis (18.3\%). More importantly, the performance curves for all three categories of etiology, including choledocholithiasis, malignancy, and benign biliary stricture, were excellent (area under the receiver operative curve $1.00,0.91$, and 0.93 , respectively). Prior studies have reported pathologic diagnosis by EUS from 6-33\% in patients with 
dilated ducts and normal liver function tests (LFTs) compared to $51-100 \%$ in patients with abnormal LFTs. ${ }^{6-9}$ The authors in this study noted that the majority of patients were symptomatic and/or had elevated LFTs and suggested that this may account for the discrepancy between their finding of malignancy as the most common etiology and choledocholithiasis seen in previous studies. ${ }^{6-9}$ Additionally, the authors demonstrated that predictive factors of a pathological diagnosis by EUS include male sex, associated intrahepatic ductal dilation, and alanine transaminase and alkaline phosphatase levels greater than the $>3$ upper limit of normal. However, further validation of these risk factors is required.

Other reasons for the variations in the diagnostic yield of EUS for the evaluation of unexplained CBD dilation among different studies include factors such as differences in the initial imaging modality used in biliary tree evaluation. Diagnostic accuracy varies among TUS, CT, and MRCP demonstrated by studies which report the significant ranges of differences between asymptomatic and symptomatic patients, as well as abnormal and normal LFTs. These differences in baseline patient characteristics may also account for the differences in diagnostic yield.

Regardless of the presence of symptoms or abnormal LFTs, the diagnostic value of EUS in the setting of an unexplained $\mathrm{CBD}$ appears to be higher than the risks incurred with more invasive diagnostic methods such as ERCP or surgery. The results of this study confirm that EUS should have a definitive place in the algorithm for the evaluation of CBD. Further prospective, comparative data are needed to determine whether EUS would provide benefit in all cases or if there are categories of patients who may benefit from proceeding directly to the intended treatment. While the sub-analysis in this study might suggest that some patients may be able to forgo EUS, a recent systematic review reported that up to $11.2 \%$ of asymptomatic patients were still found to have pathology on EUS. ${ }^{1}$ Given the low risk associated with EUS, it is reasonable to consider EUS in this patient population to define further diagnostic and therapeutic management strategies. Further cost-effective analyses would also be helpful in fine-tuning this role.

\section{Conflicts of Interests}

Amrita Sethi is a Consultant by Boston Scientific, Olympus, Fujifilm,
Medtronic, Intuitive Surgical, and Interscope. The other author has no potential conflicts of interest.

Funding

None.

Author Contributions

Conceptualization: Seifeldin Hakim, Amrita Sethi

Writing-original draft: $\mathrm{SH}$, AS

Writing-review\&editing: $\mathrm{SH}$, AS

ORCID

Seifeldin Hakim

https://orcid.org/0000-0001-7898-9611

Amrita Sethi

https://orcid.org/0000-0001-7989-0242

\section{REFERENCES}

1. Chhoda A, Dawod S, Grimshaw A, Gunderson C, Mahadev S. Evaluation of diagnostic yield of EUS among patients with asymptomatic common bile duct dilation: systematic review and meta-analysis. Gastrointest Endosc 2021;94:890-901.e8.

2. Skordilis P, Mouzas IA, Dimoulios PD, Alexandrakis G, Moschandrea $\mathrm{J}$, Kouroumalis E. Is endosonography an effective method for detection and local staging of the ampullary carcinoma? A prospective study. BMC Surg 2002;2:1.

3. Nandalur KR, Hussain HK, Weadock WJ, et al. Possible biliary disease: diagnostic performance of high-spatial-resolution isotropic 3D T2-weighted MRCP. Radiology 2008;249:883-890.

4. Kondo $\mathrm{S}$, Isayama $\mathrm{H}$, Akahane $\mathrm{M}$, et al. Detection of common bile duct stones: comparison between endoscopic ultrasonography, magnetic resonance cholangiography, and helical-computed-tomographic cholangiography. Eur J Radiol 2005;54:271-5.

5. Pausawasdi N, Hongsrisuwan P, Kamani L, Maipang K, Charatcharoenwitthaya $P$. The diagnostic value of endoscopic ultrasonography for common bile duct dilatation without identifiable etiology detected from cross-sectional imaging. Clin Endosc 2022;55:122-127.

6. Bruno M, Brizzi RF, Mezzabotta L, et al. Unexplained common bile duct dilatation with normal serum liver enzymes: diagnostic yield of endoscopic ultrasound and follow-up of this condition. J Clin Gastroenterol 2014;48:e67- e70.

7. Malik S, Kaushik N, Khalid A, et al. EUS yield in evaluating biliary dilatation in patients with normal serum liver enzymes. Dig Dis Sci 2007;52:508-512

8. Rana SS, Bhasin DK, Sharma V, Rao C, Gupta R, Singh K. Role of endoscopic ultrasound in evaluation of unexplained common bile duct dilatation on magnetic resonance cholangiopancreatography. Ann Gastroenterol 2013;26:66-70.

9. D'Souza LS, Buscaglia JM. The use of endoscopic ultrasound in the evaluation of unexplained biliary dilation. Gastrointest Endosc Clin N Am 2019;29:161-171 